\title{
Possibilities for the Detection of Santilli Neutroids and Pseudo-protons
}

\author{
Victor de Haan \\ Bon Physics B.V., Laan Van Heemstede, Puttershoek, The Netherlands
}

\section{Email address:}

victor@bonphysics.nl

To cite this article:

Victor de Haan. Possibilities for the Detection of Santilli Neutroids and Pseudo-protons. American Journal of Modern Physics. Special Issue: Issue II: Foundations of Hadronic Mechanics. Vol. 5, No. 2-1, 2016, pp. 131-136. doi: 10.11648/j.ajmp.2016050201.17

Received: July 8, 2015; Accepted: July 9, 2015; Published: June 1, 2016

\begin{abstract}
Following systematic mathematical, theoretical and experimental studies on the synthesis of the neutron from hydrogen, R. M. Santilli noted delayed neutron detections following the termination of tests, and attempted to represent them with the hypothesis of a new state of the hydrogen with spin zero called neutroid consisting of a proton and an electron at $1 \mathrm{fm}$ mutual distance in singlet coupling. More recently, Santilli predicted the possible existence of a second new particle called pseudo-proton characterized by the synthesis of the electron with the neutron, therefore resulting in a negatively charged unstable particle with a mean life expected in the range of that of the neutron and a mass of the order of the hydride ion. Subsequently, Santilli has indicated that, in the event confirmed, the pseudo-proton could eliminate the Coulomb barrier for nuclear syntheses and trigger nuclear transmutations with large release of heat without neutron emission, thus identifying a possible novel use of hydrogen for the industrial production of a basically new clean nuclear energy. In view of the latter possibility, in this paper specific experiments are proposed for the verification or denial of the existence of Santilli neutroid and pseudo-proton and, in case of confirmation, accurate measurements of their characteristics and production in numbers sufficient for industrial application.
\end{abstract}

Keywords: Pseudo-proton, Low Energy Nuclear Transmutation, Hydrogen Energy Source, Neutron, Pseudo-protonium, Detection, Spectroscopy

\section{Introduction}

Recently, Santilli et al. called for the experimental verification of the so-called pseudo-proton [1]. The pseudoproton is a particle predicted by Santilli [2] based on the hadronic mechanics he has derived since the 1970's (for an overview see $[3,4])$. The pseudo-proton is expected to play an important role in the upcoming field of low energy nuclear transmutations due to its ability to penetrate the atomic core with relative ease due to its negative charge [5]. It is expected that it plays a crucial role in the synthesis of hydrogen ions [6], [7]. Hence the experimental detection of the pseudo proton is important for future development of hydrogen energy.

\section{Synthesis of Neutron from Hydrogen}

The synthesis of the neutron from the hydrogen inside a star was proposed in 1920 by H. Rutherford [8], experimentally established in 1932 by J. Chadwick [9] and formalized by E. Fermi [10] via the familiar reaction

$$
p^{+}+e^{-} \rightarrow n+v
$$

The properties of proton, electron and neutron are well known and a summary is given in table 1 . The rest mass of the neutron, $n$ is $0.782 \mathrm{MeV}$ larger than the sum of the rest energies of both proton and electron, so that this reaction can only occur in sufficiently strong fields, for instance in the inside of stars or in the arc of a very strong discharge [1]. In 1978, R. M. Santilli [11] pointed out that quantum mechanics is not applicable to the above neutron synthesis because the rest energy of the neutron is $0.782 \mathrm{MeV}$ bigger than the sum of the rest energies of the proton and the electron, as shown by the energy of the rest mass for proton, electron and neutron respectively $E_{p}=938.272 \mathrm{MeV}, E_{e}=0.511 \mathrm{MeV}$ and 
$E_{n}=939.565 \mathrm{MeV}$,

$$
E_{n}-\left(E_{p}+E_{e}\right)=0.782 \mathrm{MeV}>0
$$

thus requiring a 'positive binding energy' with a resulting 'mass excess' under which the Schrödinger equation no longer admits physically consistent solutions.

In order to explain neutron synthesis, Santilli [11] proposed the construction of a non-unitary covering of quantum mechanics under the name of Hadronic Mechanics. However, non-unitary theories on a conventional Hilbert space over a conventional numeric field are known to violate causality. Only following the achievement of sufficient mathematical and theoretical maturity (for more details see [5]), while visiting in 1991 the ICTP in Trieste, Italy, Santilli [12] achieved the first known, non-relativistic, exact representation of all characteristics of the neutron in its synthesis from the hydrogen in the core of a star. A relativistic representation was achieved in 1992 when visiting the JINR in Dubna, Russia [13].

Laboratory synthesis of the neutron from a hydrogen gas was achieved in the 1960s by the Italian priest-physicist Don Carlo Borghi and his associates [14] via a metal chamber containing hydrogen at pressure traversed by microwaves and an electric discharge used to keep hydrogen ionized.

Santilli could not account for the synthesis of the neutron via microwaves. However, the same formulations predicted the laboratory synthesis of the neutron under an electric discharge suitable to force the penetration of the electron within the proton following their strong Coulomb attraction (according to Rutherford [8]) and provide $0.782 \mathrm{MeV}$ needed for the synthesis according to equation (2). Extensive tests apparently confirmed the laboratory synthesis of the neutron according to the predictions of Santilli's work [15]. Thereafter, Santilli continued systematic experimental research on the laboratory synthesis of the neutron from a hydrogen gas at the laboratory of the Institute for Basic Research, Palm Harbor, Florida (see the extensive data in Ref. [16]).

Systematic experimentations and industrial realizations are now conducted by the U. S. publicly traded company Thunder Energies Corporation (TEC) [17], which is currently organizing the production and sale of a Thermal Neutron Source (TEC domain names and patents pending) essentially consisting of a pressure vessel containing commercially available hydrogen traversed by a suitable discharge and control of hydrogen pressure, electric power and other characteristics to obtain the desired neutron flux output (see corporate paper [18] and movie [19] on a pre-production prototype.)

A recent technical review of the synthesis of the neutron has been provided by U. Abundo [20]; a review recommended to the general physics audience was provided in 2006 by the late J. V. Kadeisvili [21]; while a general review of the various mathematical, theoretical and experimental aspects has been provided in 2011 by I. Gandzha and the late J. V. Kadeisvili [22].

\section{Neutroid and Pseudo-proton}

Essential the bases for the prediction of the pseudo-proton is the natural assumption that mathematical point particles do not exist in nature. In real life particles will have a finite size and there is a finite probability that particles will overlap. When particles overlap, the intrinsic properties of the particles can change and can recover when they are released again in vacuum. A more elaborate overview is given in [22]. Hence, when a hydrogen atom consisting of a proton, $\mathrm{p}^{+}$and an electron, $\mathrm{e}^{-}$is sufficiently compressed (so that its radius is of the order of $1 \mathrm{fm}$ ) they can form a neutron via the above described procedure.

Table 1. Properties for several particles. For the pseudo-proton the range in which the property is expected is indicated. ${ }^{1}$ in units of $1.602177 \cdot 10^{-19} \mathrm{C}$.

\begin{tabular}{|c|c|c|}
\hline Name & Sym & Description \\
\hline Electron & $e^{-}$ & Elementary particle \\
\hline Proton & $p^{+}$ & Elementary particle \\
\hline Neutron & $n$ & $\begin{array}{l}\text { Elementary particle (standard model) or } \\
\text { bound proton and electron (Santilli) }\end{array}$ \\
\hline Hydrogen atom & $H$ & Proton + orbit electron \\
\hline Hydrogen ion & $\mathrm{H}_{2}^{+}$ & Ionized hydrogen molecule \\
\hline Pseudo-proton & $\tilde{p}^{-}$ & Synthesis of neutron and electron (Santilli) \\
\hline Hydride ion & $H^{-}$ & Proton +2 electrons in orbit \\
\hline Anti-proton & $p^{-}$ & Anti particle of proton \\
\hline
\end{tabular}

\begin{tabular}{lllll}
\hline & Charge $^{1}$ & $\begin{array}{l}\text { Mass } \\
10^{-27} \mathrm{~kg}\end{array}$ & $\mathrm{MeV}$ & $\begin{array}{l}\text { Magnetic mom. } \\
10^{-26} \mathrm{~J} / \mathrm{T}\end{array}$ \\
$\mathrm{e}^{-}$ & -1 & 0.00091 & 0.511 & 928.477 \\
$\mathrm{p}^{+}$ & 1 & 1.67262 & 938.272 & 1.410608 \\
$\mathrm{n}$ & 0 & 1.67493 & 939.565 & 0.966237 \\
$\mathrm{H}$ & 0 & 1.67353 & 938.783 & \\
$\mathrm{H}_{2}^{+}$ & -1 & 3.34615 & 1877.055 & \\
$\tilde{p}^{-}$ & -1 & $1.672 \ldots 1.677$ & $937 \ldots 941$ & $\approx 1$ \\
$\mathrm{H}^{-}$ & -1 & 1.67444 & 939.294 & \\
$\mathrm{p}^{-}$ & -1 & 1.67262 & 938.272 & 1.410608 \\
\hline
\end{tabular}

Both tests by Don Borghi [14] and Santilli [15,16] indicated the existence of nuclear transmutations that could solely be explained via the absorption of neutrons or 'neutron-type' particles. Santilli conducted extensive studies of this occurrence based on the detection of neutron counts that were delayed for up to 15 minutes following the termination of the tests.

These delayed neutron counts occurred under certain characteristics of the reactor, such as power, hydrogen pressure and others, insufficient to provide $0.782 \mathrm{MeV}$ needed for the synthesis of the neutron according to equation (2). By contrast, neutrons were detected under sufficient power, hydrogen density and other characteristics, to such an extend to require various evacuations of the laboratory.

In this way, Santilli proposed the possible existence of a new particle called neutroid [16] with essentially the characteristics of the neutron except having spin zero which is created by the reaction

$$
p^{+}+e^{-} \rightarrow \widetilde{n}
$$

under insufficient conditions to achieve the full reaction according to equation (1). A number of nuclear 
transmutations caused by the absorption of neutroids could then allow a quantitative representation of the delayed detection of neutrons (see Ref. [16] for brevity).

Abundo [20] noted that, in the event confirmed, Santilli neutroids would not experience the notorious Coulomb barrier opposing nuclear syntheses and can, therefore, trigger nuclear transmutations with large release of heat without the emission of neutrons, such as

$$
{ }^{1} \mathrm{H}+{ }^{7} \mathrm{Li}+\mathrm{TR} \rightarrow{ }^{7} \mathrm{Li}+\tilde{n} \rightarrow{ }^{8} \mathrm{Li} \rightarrow{ }^{8} \tilde{\mathrm{Be}}+\beta^{-} \rightarrow 2{ }^{4} \mathrm{He}+\Delta E
$$

where $\Delta E>\approx 15.3 \mathrm{MeV}$ per ${ }^{7} \mathrm{Li}$ nucleus and TR represents Santilli's trigger, namely, a mechanisms triggering the transmutation, such as instantaneous increase of power or pressure. Note that the symbol ${ }^{1} \mathrm{H}$ in the above reaction represents the hydrogen atom. Therefore, to our best knowledge, Abundo's transmutation [20] is the first in scientific records in which the hydrogen atom, rather then the proton, triggers a nuclear transmutation, thus stimulating potential new technologies centrally based on hydrogen.

To understand the occurrence, one should note that Santilli neutroid is essentially a new (unstable) form of the hydrogen atom with the proton and electron in singlet coupling kept together by the very strong Coulomb attraction at $1 \mathrm{fm}$ mutual distance. Santilli's trigger is, therefore, a mechanism essentially triggering the transition from the hydrogen to the neutroid that, as such, has a number of possible engineering realizations.

Following the systematic studies on the synthesis of the neutron from hydrogen, Santilli predicted in the Appendix of Ref. [2] the possible existence of a second new particle under the name of pseudo-proton with symbol $\widetilde{p}^{-}$, generated by the synthesis of the electron, this time, with the hydrogen atom, according to the reaction

$$
\left(p_{\downarrow}^{+}+e_{\uparrow}^{-}\right)+e_{\downarrow}^{-} \rightarrow \tilde{p}^{-}
$$

Note that, in comparison with Fermi's reaction (equation (1)), Santilli's reaction does not require the emission of a neutrino. The pseudo-proton will have a spin $1 / 2$, a negative unit charge, essentially the same charge radius and rest energy of the proton, and a mean life (when isolated) of the order of that of the neutron. The properties of the pseudoproton are summarized in table 1 . It is expected that the magnetic moment is of the same order as that of the proton and neutron as the charge radius and rest energy are also of the same order. It will be determined by the precise shape and velocity of the constituting charge distribution. The charge and mass of the pseudo-proton will be similar to that of the anti-proton or hydride ion.

More recently, Santilli [5] indicated that, in the event confirmed, the pseudo-proton would not only eliminate the Coulomb barrier for nuclear syntheses, but could actually be attracted by nuclei, thus triggering esoenergetic nuclear transmutations without neutron emission and significantly increase the possibilities for the industrial development of a basically new and clean nuclear energy [5] (TEC domain names and patents pending).

In order to illustrate the significance of the possibility, Santilli indicated the possible triggering by pseudo-protons of nuclear transmutations, such as [5] the transmutation of Li-7

$$
\tilde{p}^{-}+{ }^{7} \mathrm{Li} \rightarrow{ }^{8} \mathrm{~L} i+\beta^{-} \rightarrow{ }^{8} \mathrm{Be}+\beta^{-} \rightarrow 2^{4} \mathrm{He}+\beta^{-}+\Delta E_{1}
$$

with $\Delta E_{1}=2.887 \cdot 10^{-12} \mathrm{~J}$, or Pd-106

$$
\tilde{p}^{-}+{ }^{106} P d \rightarrow \tilde{}{ }^{107} P d+\beta^{-} \rightarrow{ }^{107} A g+\beta^{-}+\Delta E_{2}
$$

or Au-197

$$
\tilde{p}^{-}+{ }^{197} A u \rightarrow{ }^{198} A u+\beta^{-} \rightarrow{ }^{198} A u+\beta^{-}+\Delta E_{3}
$$

where Au-198 is unstable and decays in 2.69 days into betas and $\mathrm{Hg}-198$ with the release of $1.372 \mathrm{MeV}$, and others, all releasing a rather significant amount of heat, none of them emitting neutrons, while beta emission being easily trapped by a metal shield.

In summary, the above studies indicate possible, basically new and clean nuclear energies centered on the use of hydrogen, and its processing via suitable reactors first into neutroids and/or pseudo-protons and then their use to trigger esoenergetic nuclear transmutations. Due to the evident significance of these studies, both per se, as well as with respect to the emerging new hydrogen era, in this paper experiments are studied aimed at the confirmation or denial of the existence of Santilli neutroids and pseudo-protons and, in case affirmative, reach accurate measurements of their characteristics and their production in number sufficient for industrial applications. It should be noted that in this paper the possible connection between Santilli pseudo-proton and the anti-protons produced at various laboratories is ignored, since such a topic has been preliminarily studied in Refs. [2,5], although the technologies for the production of the two particles can benefit each other, thus warranting additional studies.

From the above it is clear that it is expected that neutrons and pseudo-protons will be produced in a sufficiently strong discharge in hydrogen gas as has been performed by Santilli [1]. That neutrons can be produced in hydrogen gas is well known and experimentally verified by many experiments [23-26] and developed for industrial applications [27]. In general it is assumed that neutrons are produced as a byproduct of the fusion of two protons, deuterons, tritons or their combinations resulting in the production of neutrons with high kinetic energies, so-called fast neutrons. The discharge acts as a means to accelerate the hydrogen ions to such high energies that the Coulomb repulsion can be overcome so that the fusion reaction can occur. However, the energy needed to overcome the Coulomb repulsion so that two ions can come as close to each other as several femtometer (the size of the proton, or range of the strong 
force) is of the order of $1.4 \mathrm{MeV}$ which is much larger than the kinetic energy available. As a recourse for this problem it is assumed that quantum tunneling exists. In that case the ion nuclei need to overlap and the tunneling probability will be large enough to give a reasonable yield. In such a case also the possibility for the above mentioned reactions exists and neutron or pseudo-proton production can occur.

\section{Detection Possibilities}

In the above it has been made clear that it is possible that neutrons or pseudo-protons can be created in a hydrogen gas discharge. In the following it is discussed how these particle might be detected.

\subsection{Neutrons}

Neutrons are neutral and have limited interaction with most materials. One should differentiate between fast neutrons that are created during fusion of fission reactions and thermal neutrons that are created by the Santilli synthesis or by moderation (i.e. slowing down) of fast neutrons. The kinetic energy of fast neutrons is of the order of $\mathrm{MeV}$ while the kinetic energy of thermal neutrons is of the order of several tens of meV. When fast neutrons have collisions with cores of the material constituting atoms or molecules, part of their energy will be released to the core and the neutrons energy decreases. After many collisions the fast neutron is slowed down to a speed comparable to the speed of the atoms or molecules of the material. At room temperature this corresponds to an average kinetic energy of $25 \mathrm{meV}$. Only after the neutrons are slowed down their energy is low enough to be absorbed during the interaction with the nucleus.

Thermal neutrons are detected by a nuclear absorption process inside the detector. The nuclear absorption creates a charged particle pair that is detected by means of an appropriate mechanism. This can be an avalanche detector in case of a gas-filled detector like a ${ }^{3} \mathrm{He}$ or $\mathrm{BF}_{3}$ detector. This can also be a photo-multiplier in case of a scintillator detector like a LiF crystal or Li-glass. The key point is that one can discriminate between fast and slow neutrons by means of a time-of-flight technique or by means of an appropriate thermal neutron absorbing material or fast neutron moderator.

The time-of-flight technique is based on the measurement of the arrival time of the neutron in the detector after it has been created. During this time the neutron has to travel the known distance from source to detector. As the travel time and travel distance are measured, the velocity of the neutrons can be determined. Slow neutrons will travel with speeds of the order of $1 \mathrm{~km} / \mathrm{s}$ while fast neutrons will travel with speed of at least $10000 \mathrm{~km} / \mathrm{s}$. To determine the travel time it is needed to know when the neutrons are emitted form the source. One method is by using a pulsed source so that the time interval in which the neutrons are created is known.

When the source is surrounded by a thermal neutron absorber (like for instance Cadmium, Gadolinium, Lithium or Boron) no thermal neutrons can escape the source. Then any excess neutron detected by the detector after turning on the source is due to the moderation of fast neutrons created in the source. When the source is also surrounded by a fast neutron moderator the number of excess neutrons in the detector will increase as more neutrons will be moderated. With this scheme it is possible to determine a) whether or not neutrons are created and b) whether fast neutrons or slow neutrons are created and c) when slow neutrons are created a indication of their energy distribution can be measured. The cost for a time-of-flight detection including the needed materials for absorption and moderation is estimated between 20 and $50 \mathrm{kUSD}$.

\subsection{Pseudo-protons}

Pseudo protons are negatively charged particle with a mass comparable with that of the hydrogen atom or proton. It can be discriminated from an electron in a mass spectrometer due to the large mass difference and from a proton because of its reversed charge. In a hydrogen discharge also hydride ions will be produced. The mass and charge of the hydride ion is comparable to that of the pseudo-proton. Hence, it is possible that already in experiments that have been performed in the past the pseudo-proton was detected, but interpreted as hydride ion [25-28]. The mass difference between the hydride ion and the pseudo-proton is expected to be very small of the order of $0.1 \%$ (see table 1 ). Hence, when using a mass spectrometer to determine what kind of particle is detected, the accuracy should be high enough to measure this small difference. An additional test might be done by measuring the magnetic moment of the detected negatively charged particle by means of an appropriate device as for example a penning trap. Mass spectrometers can be bought from the shelve, but one with an accuracy of $0.1 \%$ for a mass of approximately $1 \mathrm{amu}$ is not readily available and must be developed. In such a case it can be combined with the magnetic moment measurements. Estimated costs for such a development are between 500 kUSD and 1 MUSD.

\subsection{Pseudo-protonium Atom}

The pseudo-proton can quickly capture a proton to form a pseudo-protonium atom, $\tilde{p} p$ where the proton and pseudoproton circle around each other like in a 2-body system.

Hence, one can imagine that in a strong electrical discharge in hydrogen gas the following reaction might occur

$$
\begin{aligned}
& H_{2} \rightarrow \mathrm{H}+\mathrm{H} \rightarrow\left(p_{\downarrow}^{+}+e_{\uparrow}^{-}\right)+\left(p_{\uparrow}^{+}+e_{\downarrow}^{-}\right) \rightarrow \\
& \left(p_{\downarrow}^{+}+e_{\uparrow}^{-}+e_{\downarrow}^{-}\right)+p_{\uparrow}^{+} \rightarrow \tilde{p}_{\downarrow}^{-}+p_{\uparrow}^{+} \rightarrow \tilde{p} p
\end{aligned}
$$

Hence, in the event confirmed, pseudo-protons can form pseudo-protonium atoms during the discharge process. These atoms have energy levels determined by the energy levels of a two body system. The reduced mass of this system is about 1836 times larger than that of the hydrogen atom, comparable to those of protonium [32], resulting in energy levels in the $\mathrm{keV}$ range. These levels can be detected by means of X-ray spectrometry. The Lymann [33] (transitions form level $n>1$ 
to 1) and Balmer [33] series (transitions form level $n>2$ to

2) for this atom are shown in table 2.

The intensity of the spectral lines depends on the number density of the pseudo-protonium atoms and hence will depend strongly on the discharge parameters and the pressure of the hydrogen gas. If these series can be found in the spectra of hydrogen gas discharges with sufficient accuracy, then also the reduced mass of the pseudo-protonium atom and hence the mass of the pseudo-proton can be measured. It should be noted that in some Energy-dispersive X-ray spectroscopy experiments peaks are recorded that are close to the estimated value giving clues under what conditions pseudo-protonium might be formed [34]. The estimated costs of such an experiment are between 100 and $200 \mathrm{kUSD}$.

Table 2. The energy differences and wavelength in nm of the Lymann and Balmer series for pseudo-protonium atom.

\begin{tabular}{llllll}
\hline Lymann Transition of $\mathbf{n}$ & $\mathbf{2} \rightarrow \mathbf{1}$ & $\mathbf{3} \rightarrow \mathbf{1}$ & $\mathbf{4} \rightarrow \mathbf{1}$ & $\mathbf{5} \rightarrow \mathbf{1}$ & $\infty \rightarrow \mathbf{1}$ \\
\hline Energy difference in $\mathrm{keV}$ & 9.367 & 11.102 & 11.71 & 11.99 & 12.49 \\
Wavelength in $\mathrm{nm}$ & 0.1318 & 0.1112 & 0.1054 & 0.1030 & 0.0989 \\
\hline
\end{tabular}

\begin{tabular}{lllllll}
\hline Balmer Transition of $\mathbf{n}$ & $\mathbf{3} \rightarrow \mathbf{2}$ & $\mathbf{4 \rightarrow \mathbf { 2 }}$ & $\mathbf{5} \rightarrow \mathbf{2}$ & $\mathbf{6} \rightarrow \mathbf{2}$ & $\mathbf{7 \rightarrow \mathbf { 2 }}$ & $\mathbf{0} \boldsymbol{\infty} \rightarrow \mathbf{2}$ \\
\hline $\begin{array}{l}\text { Energy difference in } \\
\mathrm{keV}\end{array}$ & 1.735 & 2.342 & 2.623 & 2.776 & 2.868 & 3.122 \\
Wavelength in nm & 0.712 & 0.527 & 0.471 & 0.445 & 0.431 & 0.395 \\
\hline
\end{tabular}

\section{Conclusions}

Following lifelong mathematical, theoretical, and experimental studies on the synthesis of the neutron from hydrogen [19] Santilli proposed the possible existence of two new unstable particles: the neutroid [16], which is essentially a new, spin zero bound state of a proton and an electron in singlet coupling at $1 \mathrm{fm}$ mutual distance, and the pseudoproton $[2,5]$, which is characterized by the 'compression' of two electrons with opposite spins inside the proton, rather than a single 'compression' according to Rutherford [8].

By remembering that the synthesis of the neutron from the hydrogen exists in nature, and that the probability for the synthesis of the pseudo-proton is smaller yet close to that of the neutron, Santilli neutroids and/or pseudo-protons may generate basically new applications of hydrogen for industrially meaningful, and environmentally acceptable new energies. Since these possibilities are already under industrial development [34], it appears advisable to conduct joint scientific studies.

In this paper experimental means to verify or deny the existence of Santilli neutroids and pseudo-protons are described. In the affirmative case, these means can achieve accurate measurements of the particles characteristics and provide information to produce them in sufficient number to have industrial significance. Since the charge and mass of the pseudo-proton are comparable to those of the hydride ion, it is possible that in previous experiments the pseudo-proton has already been measured but not identified.

For the identification, sufficiently accurate measurements of its mass are needed (accuracy at least $0.1 \%$ ) or a measurement of the magnetic moment by means of a penning trap should be performed. It is also possible that another kind of hydrogen molecule, called a pseudo-protonium atom, is formed when a pseudo-proton and proton are in orbit around each other. When a sufficient density of pseudo-protonium is realized, it is possible to measure its Lymann and Balmer series. When this property can be measured with high accuracy, it can be used to determine the reduced mass of the pseudo-protonium atom and hence the mass of the pseudoproton.

\section{Acknowledgments}

The author would lime to thank R.M. Santilli for valuable comments and Thunder Energy Cooperation for financial support for writing this paper.

\section{References}

[1] R. Santilli, A. Nas, Confirmation of the laboratory synthesis of neutrons from a hydrogen gas, Journal of Computational Methods in Sciences and Engineering 14 (6) (2014) 405-414. URL http://www.thunderenergies.com/docs/neutron-synthesis2014.pdf

[2] R. Santilli, Apparent detection of antimatter galaxies via a refractive telescope with concave lenses, Clifford Analysis, Clifford Algebras and their Applications 3 (2014) 1-26.

[3] R. Santilli, Elements of Hadronic Mechanics, Volumes I, 1st Edition, Ukraine Academy of Sciences, Kiev, 1995. URL http://www.santilli-foundation.org/docs/Santilli-300.pdf

[4] R. Santilli, Elements of Hadronic Mechanics, Volumes II, 1st Edition, Ukraine Academy of Sciences, Kiev, 1995. URL http://www.santilli-foundation.org/docs/Santilli-301.pdf

[5] R. Santilli, Apparent nuclear transmutations without neutron emission triggered by pseudoprotons, American Journal of Modern Physics 4 (2015) 15-18. URL http://www.thunderenergies.com/docs/pseudoproton-2014.pdf

[6] M. D. Giulio, E. Filippo, D. Manno, V. Nassisi, Analysis of nuclear transmutations observed in $d$-and h-loaded pd films, International Journal of Hydrogen Energy 27 (5) (2002) 527531.

[7] T. Ohmori, T. Mizuno, H. Minagawa, M. Enyo, Low temperature nuclear transmutation forming iron on/in gold electrode during light water electrolysis, International Journal of Hydrogen Energy 22 (5) (1997) 459 - 463.

[8] H. Rutherford, Bakerian lecture: Nuclear constitution of atoms, Proceedings of the Royal Society A 97 (1920) 374.

[9] J. Chadwick, The existence of a neutron, Proceedings of the Royal Society 320 A 136 (1932) 692.

[10] E. Fermi, Nuclear Physics, University of Chicago Press, 1949.

[11] R. Santilli, Foundation of Theoretical Mechanics, Volumes I (1978) and II (1982), Springer-Verlag, Heidelberg, Germany, 1978. URL http://www.santilli-foundation.org/docs/Santilli209.pdf and http://www.santilli-foundation.org/docs/santilli69.pdf 
[12] R. Santilli, Apparent consistency of rutherford's hypothesis on the neutron structure via the hadronic generalization of quantum mechanics - $i$ : Nonrelativistic treatment, ICTP communication IC/91/47. URL http://www.santillifoundation.org/docs/Santilli-150.pdf

[13] R. Santilli, Representation of the synthesis of the neutron inside stars from the hydrogen atom. communication of the joint institute for nuclear research, dubna, russia, number jinre4-93-352 (1993), Chinese J. System Engineering and Electronics 6 (1995) 177-199. URL: http://www.santillifoundation.org/docs/Santilli-18.pdf

[14] C. Borghi, C. Giori, A. Dallolio, Evidence on the emission of neu-trons from a cold hydrogen plasma, Communications of CENUFPE Number 8 (1969) and 25 (1971), reprinted via Santilli's proposal in the (Russian) Phys. Atomic Nuclei, 56, 205 (1993).

[15] R. Santilli, Confirmation of don borghi's experiment on the synthesis of neutrons from protons and electrons, ArXiv Physics e-printsarXiv: physics/0608229. URL http://arxiv.org/pdf/physics/0608229v1.pdf

[16] R. Santilli, Apparent confirmation of don borghi's experiment on the laboratory synthesis of neutrons from protons and electrons, Hadronic Journal 30 (2007) 709-729. URL http://www.i-b-r.org/NeutronSynthesis.pdf http: //www.neutronstructure.org/neutron-synthesis-3.htm http: //www.neutronstructure.org/neutron-synthesis-2.htm

[17] P. Fleming, Thunder energies corporation, website. URL http://www.thunder-energies.com

[18] R. Santilli, A. Nas, Confirmation of the laboratory synthesis of neutrons from a hydrogen gas, Thunder Energies Corporation, 1444 Tarpon Springs, FIL 34689, U.S.A.

[19] R. Santilli, A. Nas, 12 minutes film on the laboratory synthesis of neutrons from a hydrogen gas, WORLD LECTURE SERIES ON HADRONIC MATHEMATICS, MECHANICS AND CHEMISTRY. URL http://www.world-lectureseries.org/newtron-synthesis-08-14

[20] U. Abundo, Interpretation and enhancement of the excess energy of rossi's reactor via santilli neutroids and nucleoids, Hadronic Journal 37 (2014) 697 - $737 . \quad$ URL http://www.thunder-fusion.com/docs/abundo-paper-2014.pdf $(106 \mathrm{MB})$

[21] J. Kadeisvili, The rutherford-santilli neutron, Hadronic Journal 31 (2008) 1. URL http:nwww.i-b-r.org/RutherfordSantilli-neutron.htm URL:http://www.thunderenergies.com/docs/pseudoproton-2014.pdf
[22] I. Gandzha, J. Kadeisvili, New Sciences for a New Era: Mathematical, Physical and Chemical Discoveries of Ruggero Maria Santilli, Sankata Printing Press, Nepal, 2011. URL http://www.santilli-foundation.org/docs/RMS.pdf

[23] J. Verbeke, K. Leung, J. Vujic, Development of a sealedaccelerator-tube neutron generator, Applied Radiation and Isotopes 53 (45) (2000) $801-809$.

[24] M. Saltmarsh, Warm fusion, Nature 434 (45) (2005) 1077 1078.

[25] B. Naranjo, J. Gimzewski, S. Putterman, Observation of nuclear fusion driven by a pyroelectric crystal, Nature 434 (45) (2005) 1115-1117.

[26] J. Reijonen, F. Gicquel, S. Hahto, M. King, T. Lou, K. Leung, Dd neutron generator development at $L B N L$, Applied Radiation and Isotopes 63 (56) (2005) 757 - 763, 8th International Conference on Applications of Nuclear Techniques.

[27] J. Sved, Applications for gas-plasma target neutron generators, Nuclear Research Applications and Utilization of Accelerators, Vienna, Austria, 48 May 2009.

[28] K. Ehlers, K. Leung, Multicusp negative ion source, Review of Scientific Instruments 51 (6) (1980) 721-727.

[29] M. Bacal, A. Hatayama, I. Matsumiya, M. Hamabe, T. Kuroda, Y. Oka, Extraction physics in volume h-ion sources, Review of Scientific Instruments 77 (3) (2006) 03A502.

[30] M. Bacal, Negative hydrogen ion production in fusion dedicated ion sources, Chemical Physics 398 (0) (2012) 3 - 6, chemical Physics of Low-Temperature Plasmas (in honour of Prof Mario Capitelli).

[31] P. Franzen, D. Wnderlich, U. Fantz, the NNBI Team, On the electron extraction in a large rf-driven negative hydrogen ion source for the iter nbi system, Plasma Physics and Controlled Fusion 56 (2) (2014) 025007.

[32] C. Batty, Antiprotonic-hydrogen atoms, Reports on Progress in Physics 52 (1989) $1165-1216$.

[33] F. Jenkins, H. White, Fundamentals of Optics, 11th Edition, McGraw-Hill, 1982.

[34] E. Storms, The explanation of low energy nuclear reaction, an explanation of the relationship between observation and explanation, 1st Edition, Infinite Energy Press, Concord, NH, USA, 2014 